\title{
Mechanical Energy Harvesting Taxonomy for Industrial Environments: Application to the Railway Industry
}

\author{
Pablo López Díez, Iosu Gabilondo, Eduard Alarcón, Francesc Moll
}

\begin{abstract}
Traditional industry is experiencing a worldwide evolution with Industry 4.0. Wireless sensor networks (WSNs) have a main role in this evolution as an essential part of data acquisition. The way in which WSNs are powered is one of the main challenges to face if Industry wants to achieve the digital transformation. Energy harvesting technologies are one of the possible solutions to this challenge. The main purpose of this paper is to present a novel method to taxonomize knowledge in the field of mechanical energy harvesting to enhance the use of energy harvesting technologies in industrial applications. The methodology is based on the analysis of key parameters and performance metrics for existing technologies. The taxonomy is applied to rail axles in order to select the energy harvesting technology that is more appropriate for this specific location, demonstrating the potential of mechanical energy harvesting technologies (MEHTs) for the railway industry, as a use case of industrial environment. Additionally, the taxonomy allows to identify upcoming challenges for research purposes while analyzing the compatibility among mechanical energy harvesting technologies in order to create hybrid harvesters.
\end{abstract}

\section{Index Terms}

Energy harvesting, Industrial Internet of Things (IIoT), Mechanical energy, Rotational motion, Railway industry.

\section{INTRODUCTION}

In the next few years the Industrial Internet of Things (IIoT) is expected to offer promising solutions to transform many existing industrial sectors such as transportation and manufacturing [2], consolidating the development of Industry 4.0. WSNs composed by Electronic Smart Systems (ESSs) will have a crucial role in this growth as a very important part of IIoT. In order to provide these systems with the necessary portability and energy autonomy, wireless and compact energy sources are needed [3]. Nowadays, these devices are powered using primary batteries in most cases, but the interest in energy harvesting devices is increasing [4] as the best alternative to combine with batteries.

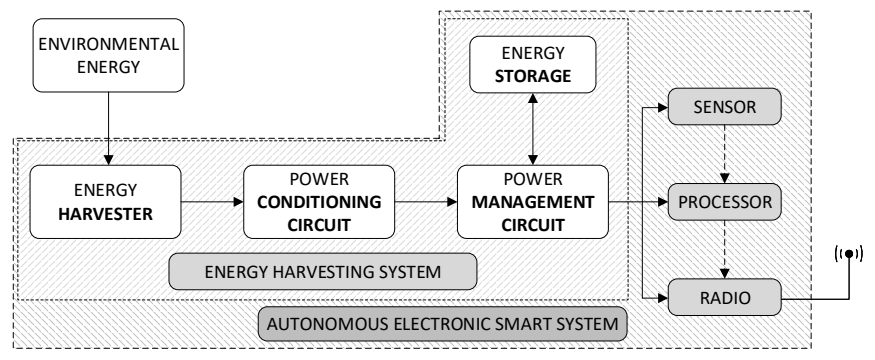

Fig. 1. Typical AESS structure

Energy harvesting systems convert different kinds of energy harvesting sources (EHSs) available in the environment into the electrical domain [5] by using energy harvesters based on energy harvesting technologies (EHTs). Electric energy is conditioned by the power conditioning circuit (PCC) to be stored into an energy storage element or delivered to the load by the power management circuit (PMC) as represented in Fig.1, which describes the structure of a typical Autonomous Electronic Smart System (AESS).

Energy harvesting in industrial environments can be classified into three categories: mechanical energy harvesting (MEH), thermal energy harvesting (TEH) and radiant energy harvesting (REH) [6], as described in Fig.2. Each category has different kinds of EHSs which are converted into electric energy through EHTs.

The number of publications in the field of energy harvesting has significantly increased in the last years [7]. Therefore, a systematic and structured review of knowledge in this field is timely and needed, in order to define a detailed energy harvesting

Pablo López Díez and Iosu Gabilondo are with the Microsystems Team, IK4-Ikerlan, 20500 Mondragón, Spain (e-mail:\{plopez,igabilondo\}@ikerlan.es)

Eduard Alarcón and Francesc Moll are with the Department of Electronic Engineering, Universitat Politècnica de Catalunya, 08034 Barcelona, Spain (e-mail:\{eduard.alarcon,francesc.moll \}@upc.edu)

Part of this work was accepted for IEEE International Symposium on Circuits \& Systems Conference (ISCAS), Florence, Italy, May. 2018 [1] 


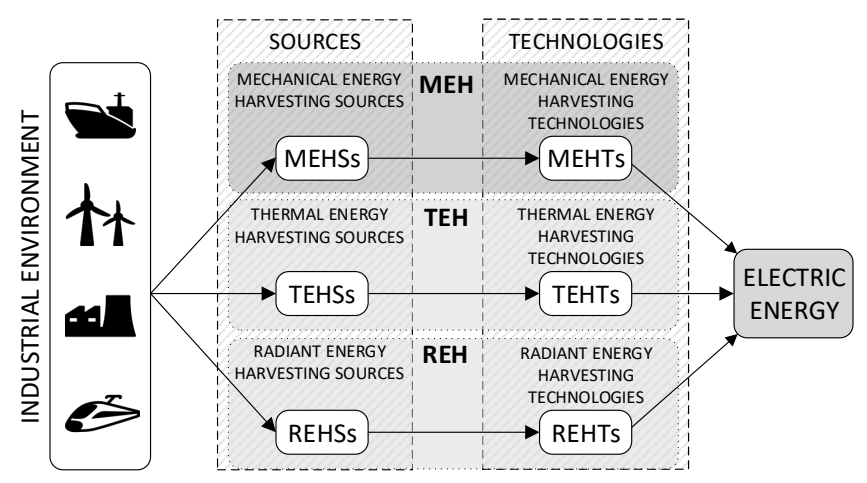

Fig. 2. Industrial Environment Energy Harvesting

knowledge structure. Previous works have studied in detail EHTs [3], [8], but a procedure to analyze the compatibility between EHSs and EHTs has not been done.

The purpose of this article is to present the guidelines to be followed in order to elaborate a comprehensive energy harvesting taxonomy, which can be used as a method to select the EHT approach that is more appropriate for each industrial location. An energy harvesting category has to be selected from all existing categories (MEH, TEH and REH) to perform the taxonomy. Since mechanical energy is the most common kind of energy in industrial environments [9], MEH have been selected for study. In particular, due to the large amount of mechanical energy sources that can be found in an industrial environment, the taxonomy is applied to the representative and challenging case of rotary locations. However, the procedure applied in this article can be used in the same way to structure the rest of MEHSs and taxa (TEH and REH). The procedure is based on the following steps:

1) Summarize the current state of the art of MEH with a systematic review of MEHSs and MEHTs (Section II-A and II-B respectively).

2) Perform a method to taxonomize MEH based on performance profiles and apply it to rotational motion (Section III-A and Section III-B respectively), identifying MEHTs trends and research and technology gaps in the field of MEH.

Once MEH taxonomy is performed, it is applied to the railway industry due to the large number of applications that WSNs have in this sector [10]. It is applied to rail axles, of the many possible locations, in order to select the MEHT that is more appropriate for this specific location, which is of strategic interest.

\section{MEH KNOWLEDGE REVIEW}

In this section, a comprehensive state of the art of MEHSs and MEHTs is presented.

\section{A. Mechanical Energy Harvesting Sources}

Mechanical energy is present in the environment in many ways such as vibration, flowing fluids or sound [11]. It is the cause of changes in an object's motion and structure. There are different types of MEHSs present in an industrial environment which have to be correctly identified in order to apply the best MEHT for each case. In the next lines a brief description of each MEHS will be given with common examples of typical locations where they can be found:

- Rotational motion: It is every motion in which each particle of a rigid object rotates around an axis, through the same angle, with the same angular speed and the same angular acceleration [12]. Examples: shafts, wheels and gears.

- Linear motion: It is that in which a body moves in a straight line. Examples: linear bearings and guides.

- Vibration: It can be found in locations where a force is applied to a body forcing it to move around its equilibrium position [12]. Examples: imbalanced mass in a system, tear and wear of materials [7] and shaft misalignment [13].

- Strain-deformation: The structure of an object can be modified in length, shape and volume by applying external forces [12] or temperature variations. Examples: industrial heat treatment and physical deformations.

- Impact: Impacts occur when two objects come together for a short period of time producing impulsive forces on each other [12]. Examples: punching machines, forging hammer machine and hydraulic breakers.

- Sound: It is every mechanical longitudinal wave that propagates through a gas, liquid or solid and the audible range of which includes frequencies from $20 \mathrm{~Hz}$ to $20 \mathrm{kHz}$ for humans [12]. Examples: industry machinery and combustion engines.

When analyzing an industrial location, it is important to consider that all MEHSs previously exposed are related to each other. Some MEHSs cause another MEHSs to be present in an industrial environment, such as vibrations in machinery which can cause deformations and sounds. Applying the appropriate MEHTs to convert secondary MEHSs in addition to primary MEHS would allow to improve the overall efficiency of the energy harvesting system. 


\section{B. Mechanical Energy Harvesting Technologies}

MEHTs convert MEHSs into electric energy. There are three main technologies based on three main physical principles of operation (piezoelectric, electromagnetic and electrostatic). The rest of MEHTs (variable reluctance, magnetostrictive, magnetoelectric and Wiegand) are considered as secondary technologies because they operate according to the above principles of operation as described in Fig.3.

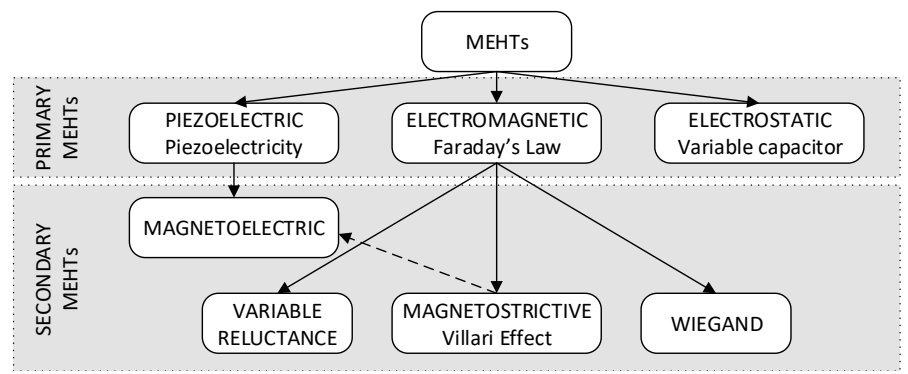

Fig. 3. MEHTs classification

1) Primary MEHTs: Primary MEHTs and their principles of operation are analyzed.

- Piezoelectric: Piezoelectric transducers generate electric power due to the ability of piezoelectric materials to exhibit a surface charge in response to applied mechanical stress [9], principle of operation known as piezoelectricity [14]. Fig.4 shows how a piezoelectric transducer produces positive/negative voltages when it is stretched/compressed (depending upon its operating mode and polarization) [15].
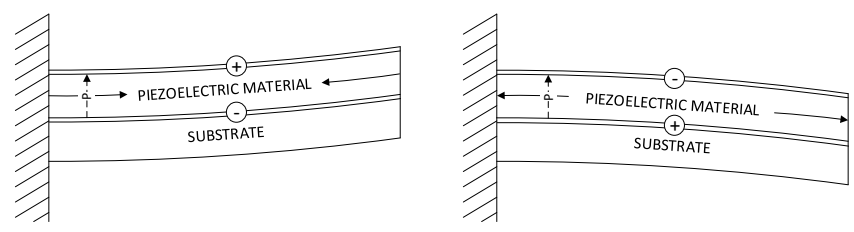

Fig. 4. Principle of operation - Piezoelectric

The output power will be higher if the system vibrates at its natural frequency [16], which dictates the selection of material (single crystals, ceramics or polymers) [17] and dimensions [7].

Piezoelectric technology is commonly used to convert vibrations into electric energy [8] but it has been also studied to convert rotational motion [18]-[25], strain-deformation [26], [27] and impacts [28]-[32] into electrical domain.

- Electromagnetic: The electromagnetic harvesters' principle of operation is based on Faraday's law of electromagnetic induction. This law states that a variable magnetic flux through a surface, bounded by the conductor, induces an electrical current in any closed circuit [9] as described in Fig.5. Electromagnetic harvesters are composed of a magnetic source and a coil that are in relative motion to each other [33].
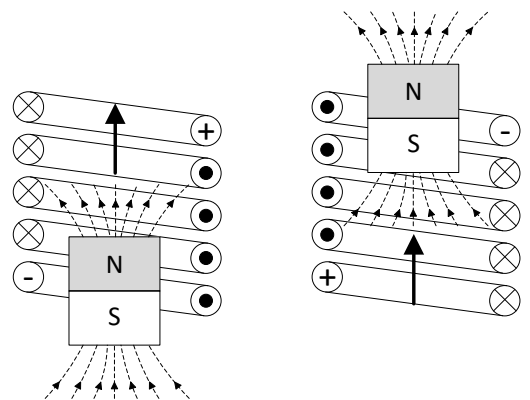

Fig. 5. Principle of operation - Electromagnetic

Electromagnetic technology is highly frequency-dependent as a consequence of Faraday's law of electromagnetic induction, as can be analyzed in eq. 1 .

$$
V(t)=-N A \frac{\mathrm{d} B}{\mathrm{~d} t} \sin (\alpha)
$$


The voltage induced by an electromagnetic harvester is proportional to the time rate of change of the magnetic field flux $(d B / d t)$ through a coil with a determined number of turns $(N)$, a determined area $(A)$ and a specific angle $(\alpha)$ between the coil area and the magnetic field flux.

Electromagnetic harvesters have been extensively analyzed to convert rotational motion and vibrations into electric energy [34], but this technology has been also studied to convert both linear motion [35] and impacts [36]-[39] into electric energy.

- Electrostatic: The electrostatic harvesters' principle of operation is based on the variable capacitor concept [40]. It is implemented by capacitive structures made of moving parallel plates. The material between these parallel plates can be air, vacuum or any dielectric material [41]. The capacitance depends on the distance $d$ between plates (contact-separation mode), the area $A$ that both plates have in common (sliding mode) and the material used in the intermediate layer as described by

$$
C_{t}=\frac{\varepsilon_{0} \varepsilon_{r} A}{d}
$$

where $\varepsilon_{0}$ is the permittivity of free space, and $\varepsilon_{r}$ is the relative permittivity of the material used in the intermediate layer [42].

Depending on these intermediate layers, electrostatic harvesters can be considered as electret-free (without intermediate material) [41], electret-based (electret material) [41] and triboelectric (two different materials -triboelectrification-) [43], which is represented in Fig.6.

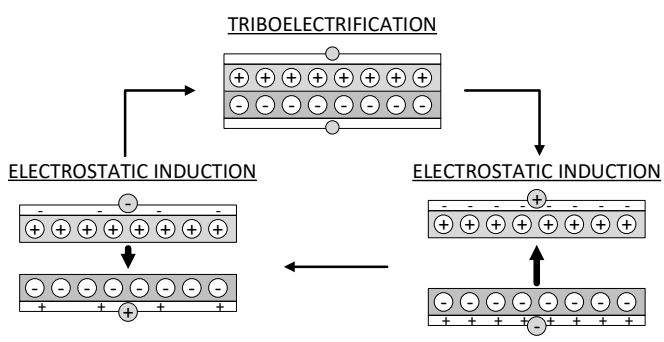

Fig. 6. Principle of operation - Electrostatic

Electrostatic technology is normally applied to harvest environmental vibrations [41] but they can be also used to harness rotational motion [44]-[49] and strain-deformation [50]-[52].

2) Secondary MEHTs: MEHTs that are based on the previous principles of operation are now analyzed.

- Variable reluctance: Variable reluctance harvesters principle of operation is based on Faraday's law described in eq. 1. In this case, the variation of magnetic field is produced as a consequence of reluctance, the term coined by Oliver Heaviside [53]. Reluctance is the analogous term of electrical resistance in the magnetic field. It forces the magnetic flux through the magnetic circuit with less reluctance. As shown in Fig.7, by modifying the reluctance of a magnetic path, a variable magnetic flux is produced and converted into electric power according to Faraday's law [54]. Variable reluctance harvesters are composed of a magnetic source, a coil (both of them stationary in relation to each other [33]) and a ferromagnetic material moving nearby that causes such reluctance variation.
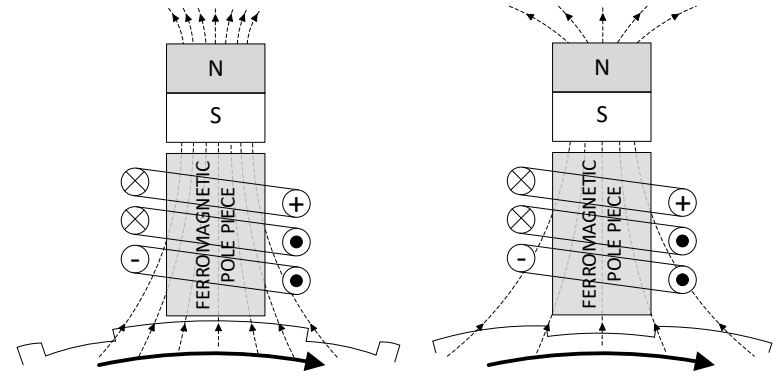

Fig. 7. Principle of operation - Variable reluctance

Variable reluctance technology is considered appropriate to convert rotational motion [33] into electric energy, but it could be also used to harness linear motion.

- Magnetostrictive: Also known as magnetoelastic harvesters, their principle of operation is based on two energy transduction principles. First energy transduction principle is the Villari effect, also known as the piezomagnetic [55] and magnetomechanical effect [56], which is used to convert mechanical domain into magnetic domain. According to the Villari effect, 
a change in magnetization of magnetostrictive materials is produced in response to applied mechanical stress [56]. These changes in magnetization are converted into electrical energy using Faraday's law, as described in Fig.8.

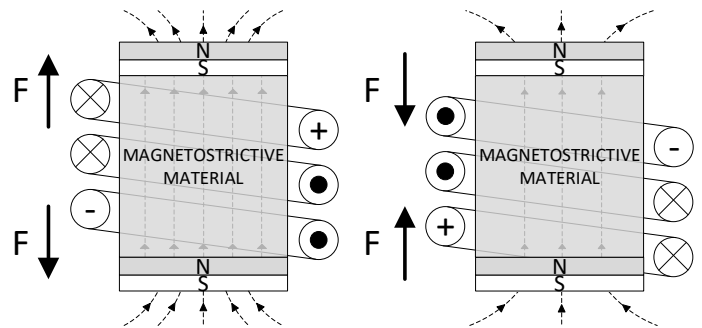

Fig. 8. Principle of operation - Magnetostrictive

A magnetostrictive harvester is composed by a magnetostrictive material (Terfenol-D, Metglas, Galfenol or Ni51.1Mn24Ga24.9) [57], a pick-up coil used to convert variable magnetic field into electric power by Faraday's law [58] and a permanent bias magnet used to induce the magnetic field [56]. The magnetomechanical interaction of magnetostrictive harvesters is expressed by the equation

$$
B=d_{33} \sigma+\mu^{\sigma} H,
$$

where $B$ is the magnetic flux density, $d_{33}$ is the parameter describing the magnetomechanical effect, $\sigma$ is the stress applied to the magnetostrictive material, $\mu^{\sigma}$ is the magnetic permeability at a constant stress and $H$ is the applied magnetic field strength [59].

The flux density $B$ variation can be converted into electric power by using Faraday's law as exposed in eq. (1).

Magnetostrictive technology is mainly used to convert vibrations [58], [60]-[62] into the electrical domain. This technology has also been analyzed to harness strain-deformation [63].

- Wiegand: Wiegand harvesters are based on J.R. Wiegand's wires invention [64]. These devices release a triangular voltage pulse with an amplitude of several volts when they are subjected to an external, time-varying magnetic field [65]. Wiegand wires have a different shell and core magnetic properties due to the patented method of manufacturing [66], which creates a hardened outer shell in the wire with a higher magnetic hysteresis $\left(H I S T_{M A X}\right)$ than the center $\left(H I S T_{M I N}\right)$ [33]. As described in Fig.9, when the external magnetic field value rises to $\left(H I S T_{M I N}\right)$, the magnetic polarization of Wiegand wire core changes producing a voltage pulse by virtue of Faraday's law. Afterwards, when the external magnetic field rises to $\left(H I S T_{M A X}\right)$, a smaller voltage pulse is produced as a consequence of the Wiegand wire shell change of magnetization. The amplitude of voltage pulses is independent of the external time-varying magnetic field frequency because the pulses are produced by changes in core and shell polarity. Thus, only the pulse rate varies with magnetic field frequency variation. This characteristic makes Wiegand transducers an attractive device for low-frequency applications [65].

Wiegand technology is generally considered appropriate to convert rotational motion into electric energy [67] but it could also be appropriate to harness linear motion.

- Magnetoelectric: Magnetoelectric harvesters use the Villari effect and piezoelectricity as energy transduction principles. They are composed by layers of magnetostrictive and piezoelectric materials [68], so that when a variable magnetic field is applied, the magnetostrictive layers are stressed thereby in turn stressing the piezoelectric layers [69]. In this way a variable magnetic field is converted into electric power by piezoelectricity as shown in Fig.10.

The voltage generated by magnetoelectric harvesters is proportional to the stress generated by the magnetostrictive layers into the piezoelectric material, which can be calculated as follows [70]

$$
\sigma_{31 p}^{E}=\frac{2 E_{m} E_{p} t_{m} \triangle \varepsilon_{0}}{(1-\nu)\left(2 E_{m} t_{m}+E_{p} t_{p}\right)},
$$

where the subscript $m$ and $p$ means magnetostrictive and piezoelectric respectively. The parameters $\sigma_{31 p}^{E}, E, t, \triangle \varepsilon_{0}$ and $\nu$ are the compressive stress in the piezoelectric layer, the elastic modulus, thickness, the linear strain of the magnetostrictive layer and Poisson's ratio respectively. Finally, the output voltage obtained from the magnetoelectric harvester is expressed as follows [70]

$$
V_{\text {OUT }}=2 \cdot g_{31} \cdot t_{p} \cdot \sigma_{31 p}^{E},
$$

where $g_{31}$ is the piezoelectric voltage constant.

This technology is generally considered appropriate to convert vibrations into electric energy [71]-[74] but it has been also analyzed to convert rotational motion into the electrical domain [75], [76]. 

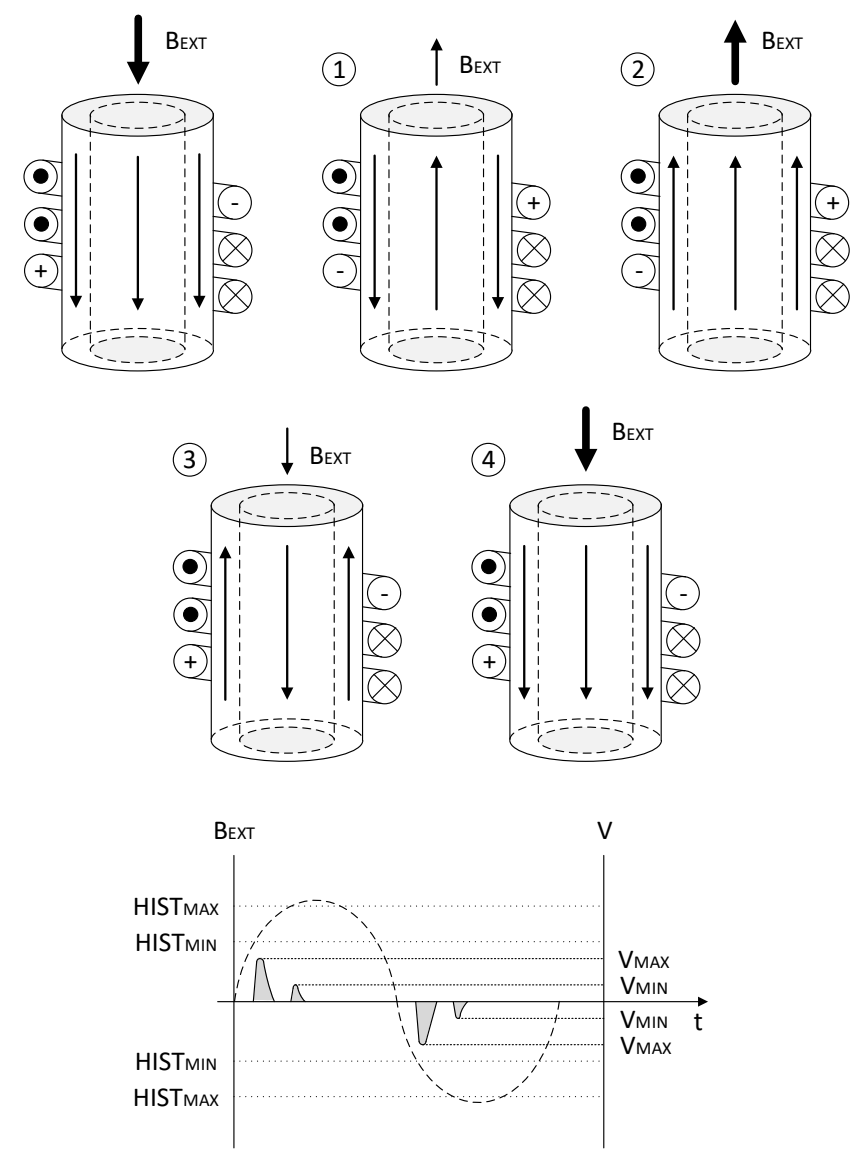

(1) (2) (3) (4)

Fig. 9. Principle of operation - Wiegand
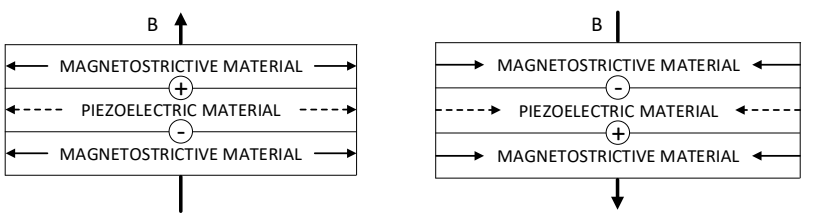

Fig. 10. Principle of operation - Magnetoelectric

\section{MEH TAXONOMY}

Once MEH systematic review has been accomplished, a method to taxonomize MEH knowledge is presented and is applied to rotational motion as MEHS. The purpose of such taxonomy is to study the relationship and capture the interplay between MEHSs and MEHTs while discovering energy harvesting trends and research and technology gaps, thereby pointing upcoming challenges. It can be also used to analyze the compatibility among MEHTs in order to create hybrid harvesters.

\section{A. Description of the method}

The method is based on key parameters analysis for each MEHT using associated bibliography for this purpose. Parameters analyzed and assessment criteria are described below:

- Robustness: The ability of each MEHT to withstand harsh conditions of industrial applications is a limiting factor in the selection of the MEHT that is more appropriate for each industrial location. It is evaluated from 1 to 5 depending on each MEHT capability to resist harsh environments (e.g. vibrations, dust and impacts).

- Miniaturization: The ability of a technology to reduce its dimensions while maintaining performance is an important parameter for the design of energy harvesting systems which are commonly used to power ESSs located in confined spaces difficult to access. It is also evaluated from 1 to 5 depending on MEHTs capability to be miniaturized.

- Frequency: In an industrial location where a wide range of frequencies can be harvested, from low frequency/high amplitude to high frequency/low amplitude excitations [77], the analysis of the frequency spectrum that each MEHT is 
capable to convert into electrical energy is an essential parameter in order to select the most appropriate technology for each industrial application.

- Acceleration: As explained in [77], the maximum power that can be harvested from a rotary source depends on its acceleration. Thus, the range of accelerations that each MEHT is capable of converting into electrical energy is a key parameter in order to optimize the energy harvesting system design.

- Number of publications: This bibliometric parameter is analyzed as an indicator of research gaps and upcoming challenges. It is evaluated according to the number of publications that evaluate each MEHT applied to each MEHS.

Other parameters of high technological interest, discarded for various reasons when developing the taxonomy, are the following:

- Technology readiness level (TRL): A metric that evaluates the maturity of a MEHT is essential to analyze the fit of this technology in a specific industrial application. However, the bibliography used to develop this taxonomy does not analyze this parameter, so it cannot be parameterized.

- Scalability: The way in which a MEHT scales not only in size but also in power is a very relevant parameter when

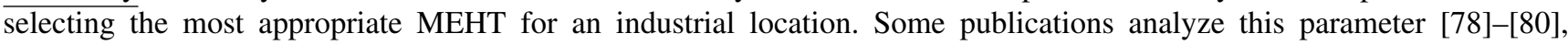
however more in-depth research is needed, so its analysis is postponed for future publications.

- Manufacturing costs: From an industrial point of view, the cost of manufacturing an energy harvesting solution is a key parameter to analyze the feasibility of a hypothetical commercial device. Nevertheless, MEHT manufacturers do not even provide cost estimates, so this parameter cannot be used to taxonomize MEH knowledge.

The selected parameters previously described are evaluated and represented in a performance profile for each MEHT as can be seen in Fig.11. The values represented in these performance profiles are therefore not the theoretical limits of each MEHT, but are the maximum values observed in the bibliography.

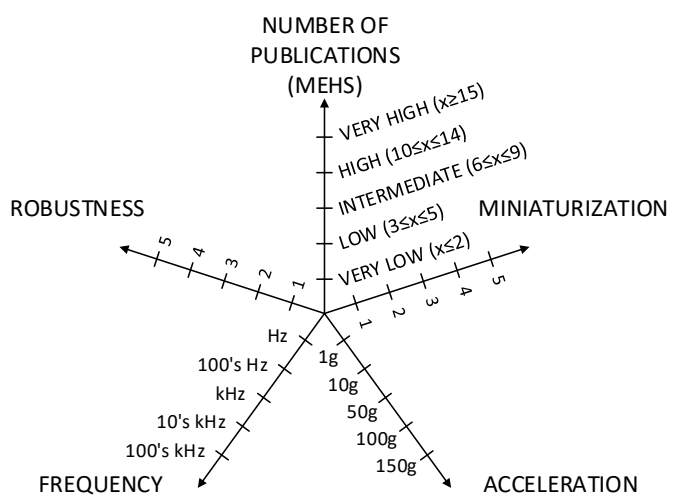

Fig. 11. MEHTs performance analysis

\section{B. Rotational motion MEH taxonomy}

In order to apply the previously exposed methodology to taxonomize, both primary MEHTs and secondary MEHTs are taxonomized for rotational motion as MEHS. It should be noted that rotational motion sometimes produces other MEHSs as secondary sources such as vibrations or impacts. Their performance profiles, represented in Fig.12 and Fig.13 respectively, have been determined based on existing bibliography [20], [22]-[25], [42], [44], [46]-[49], [79], [81]-[93].

1) Primary MEHTs performance profiles: Primary MEHTs are taxonomized according to parameters previously exposed, representing their performance profiles in Fig. 12.

- Piezoelectric: There are different ways to implement a piezoelectric harvester into a rotational motion to convert it into electricity, which are basically divided into contact and contactless devices. [18], [20], [22] analyze the possibility of using rotational motion to force impacts into the piezoelectric device that are used to produce energy, with an important mechanical wear of the device as the main disadvantage. On the other hand, contactless devices are analyzed [19], [21], [23]-[25] as a good alternative to contact devices.

As it can be seen in Fig. 12, piezoelectric main characteristics are good miniaturization (4) [81] and intermediate robustness (3) for rotational applications [19], [24]. Piezoelectric technology is able to collect from low frequencies (Hz) to intermediate frequencies $(\mathrm{kHz})$ [25], although at high frequencies the efficiency of piezoelectric devices decreases [82]. Regarding maximum acceleration that piezoelectric technology is able to convert into electric energy, high values of $35 \mathrm{G}$ have been observed [83]. In the last few years, the number of publications that analyze piezoelectric technology applied to rotational motion has increased [20], [22]-[25], [94]-[96], so it can be concluded that there is a positive trend in this field. Furthermore, there is still a challenge in adapting this technology to the rotational motion, which increases the interest in this research area. 


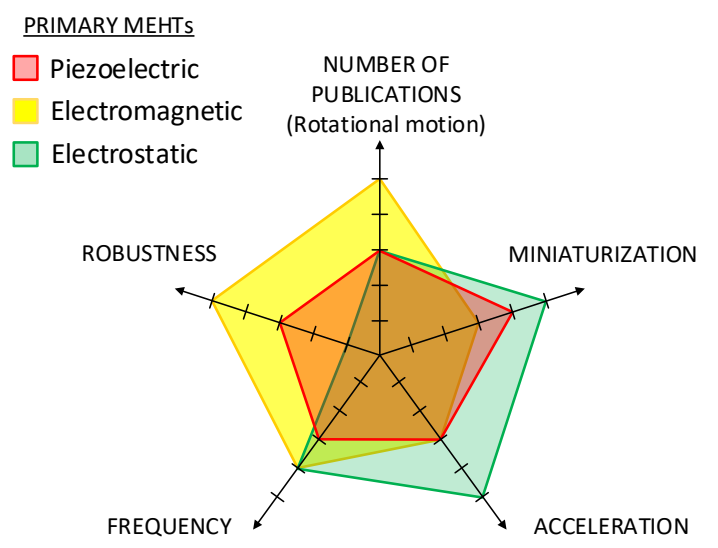

Fig. 12. Primary MEHTs performance analysis

- Electromagnetic: Electromagnetic harvesters can be placed into the rotary part of the application [88], [90], [97], [98] or into the static part [89], [91], [92], [99]. As exposed in [33], in both cases the device is composed by a magnetic source and a coil in relative motion to each other.

Electromagnetic technology's performance profile, represented in Fig. 12, shows that this technology's robustness is very high (5) as a result of having been studied for years [84]. However, their miniaturization is a critical point (3) due to their high dependence upon frequency [85] (reducing their volume would mean to increase the frequency to maintain the obtained power [79]). Electromagnetic harvesters are able to collect up to 10's $\mathrm{kHz}$ [79], [81] and accelerations of 50G [86]. Thus, electromagnetic technology is a good way to convert rotational motion into electric energy when there are no size restrictions [7]. The large number of publications available in this field [84], [88]-[92], [97], [100]-[105] indicates the interest in applying this technology to rotary locations.

- Electrostatic: The interest in electrostatic harvesters is increasing in the last years [47] as an effective way to convert rotational motion into electricity.

As it can be analyzed in Fig. 12, electrostatic converters are favorable for the miniaturization (5) [42] but they have a reduced durability and thus, a reduced robustness (1) [87]. Thus, there is an upcoming challenge to increase the durability of this technology in order to meet the robustness requirements of industrial applications. This MEHT is capable to convert high frequencies up to 10's kHz and high accelerations of 140G [81]. Regarding the publications in this field, all references that analyze the compatibility between this technology and rotational motion are focused in triboelectrification [44], [46][49], [93]. Therefore, the analysis of electret-free and electret-based electrostatic converters applied to rotary applications is a research gap to be considered.

2) Secondary MEHTs performance profiles: Similarly to primary MEHTs, secondary MEHTs' performance profiles are now analyzed.

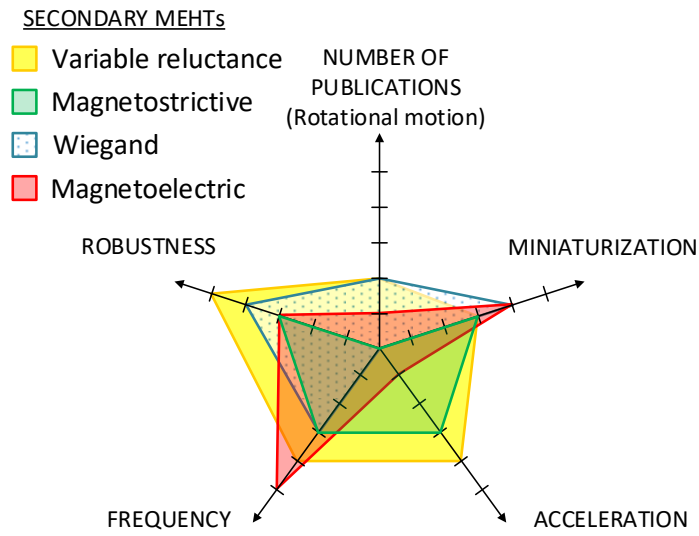

Fig. 13. Primary MEHTs performance analysis

- Variable reluctance: Depending on which structure creates the variation of reluctance, the harvester can be placed on both the rotating and the static part [106]. Variable reluctance harvesters applied to rotary locations take advantage of existent structures [54] or structures designed to optimize the variation of reluctance [107], [108] and thus the quantity of mechanical energy converted into electricity. 
As a consequence of using Faraday's law to convert rotational motion into electric energy, variable reluctance's performance profile, represented in Fig. 13, has similar characteristics to electromagnetic technology. Therefore, the robustness of this MEHT is very high (5) while its miniaturization (3) is also a critical point [109]. With regards to frequency and acceleration, variable reluctance harvesters have the same operating range than electromagnetic ones, up to 10 's $\mathrm{kHz}$ and $50 \mathrm{G}$ respectively. Analyzing the number of publications in the field of variable reluctance applied to rotational motion [33], [54], [106]-[108], it can be concluded that it is an interesting research gap to be addressed.

- Magnetostrictive: This technology has not been evaluated yet for rotational motion. It is an interesting research gap that should be explored in order to know the compatibility of magnetostrictive technology and rotary locations.

Despite of not being analyzed for rotational motion, magnetostrictive technology is characterized by a good miniaturization (3) [62] and a good robustness (3) with respect to other MEHTs [60]. Their frequency and acceleration operation limits at the present time are $1 \mathrm{kHz}$ [58], [59], [71] and 13G [110] respectively.

- Wiegand: As explained in Section II-B, Wiegand devices need a variable magnetic field to produce electric energy, so a Wiegand harvester is composed by a pair of opposite magnets in addition to a Wiegand device in order to produce changes in Wiegand's polarization. Thus, from a design standpoint, Wiegand system can be configured with a magnetic movable and Wiegand static parts or vice versa. For rotational applications, both configurations have been proposed in [111], [112] and [67] respectively.

Wiegand technology is characterized by a high robustness (4) [67] and a good miniaturization (4) [65] which make them appropriate to use in industrial environments. Wiegand devices work properly until $7 \mathrm{kHz}$ [113] and the effects of the acceleration on the power obtained are not represented in the performance profile as a consequence of not having been analyzed so far. The low number of publications in the field of rotational motion [67], [111], [112] makes Wiegand technology an opportunity to research and develop new systems.

- Magnetoelectric: No references have been found that analyze the use of magnetoelectric technology to directly convert rotational motion into electricity. However, [75], [76] study magnetoelectric technology in order to convert vibrations produced by rotational motion into electric energy by using a cantilever in the transduction process.

Despite not having been analyzed for rotary motion, magnetoelectric technology is characterized by the high capability to be miniaturized (4) [69] and its intermediate robustness (3) [74]. The maximum frequency and acceleration that magnetoelectric technology is capable to convert up to date into electric energy are $337 \mathrm{kHz}$ [69], [114] and 1G [72] respectively. The absence of articles that analyze the compatibility between magnetoelectric technology and rotational motion [75], [76] can be understood as a research opportunity that should be exploited.

\section{MEH TAXONOMY APPLIED TO RAILWAY INDUSTRY}

Once all rotary MEH knowledge has been structured in order to perform a comprehensive MEH taxonomy, it can be applied to real industry environments in order to select the MEHT approach that is more appropriate for each industrial location.

The railway industry is the selected use case as a consequence of the large number of applications that WSNs have in the railway sector, such as infrastructure and vehicle health monitoring and logistical tracking solutions [10]. There are many possible locations to place an energy harvesting system in a railway environment, depending on sensing requirements. Authors have selected three of all possible locations to analyze which kind of MEHSs are present in each one, performing a MEHSs analysis as described in Fig.14.

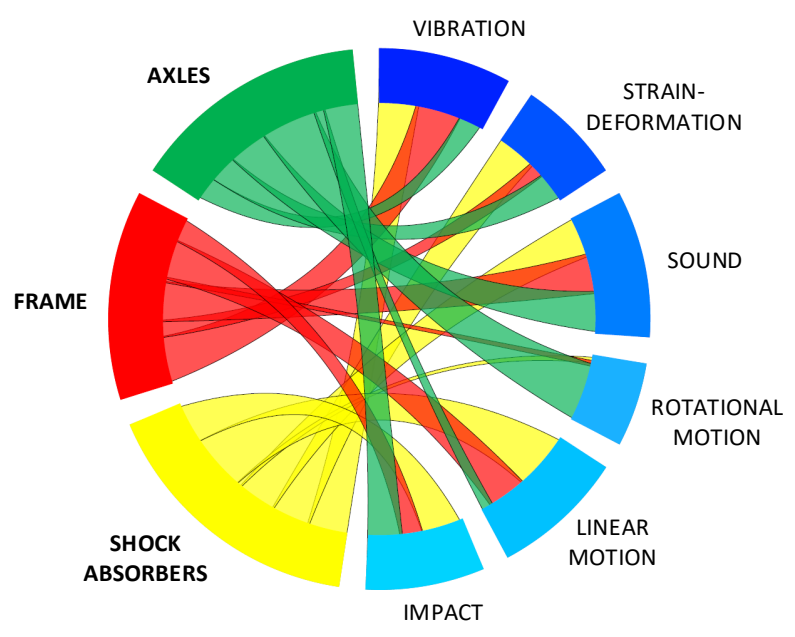

Fig. 14. Railway environment MEHSs analysis 
From all possible locations into the train and its structure, rail axles have been selected by authors to apply the MEH taxonomy. The MEHSs analysis described in Fig.14 shows that a large quantity of rotational motion can be found in this location [115]. Thus, rotational motion MEH taxonomy previously performed in Section III-B must be applied.

\section{A. Location analysis}

In order to select the MEHT that is more appropriate for rail axles using the rotational motion MEH taxonomy, an analysis of the location has to be performed. As mentioned in [115], railway environments are very harsh, especially those near the bogie and axles. Because of this reason a robust (4) MEHT must be used. There is also not much space available near the axles, so a severe space limitation is present in the use case. As a result, a MEHT with a good miniaturization (3) must be selected. Regarding rotation frequency and accelerations of rail axles, their values usually reach 100 's $\mathrm{Hz}$ and 10 's G respectively. Finally, no research publications have been found in the field of MEH applied to rail axles.

Once all these parameters have been analyzed, axles parameters profile is performed and represented in Fig.15, in order to compare it with previous MEHTs ones.

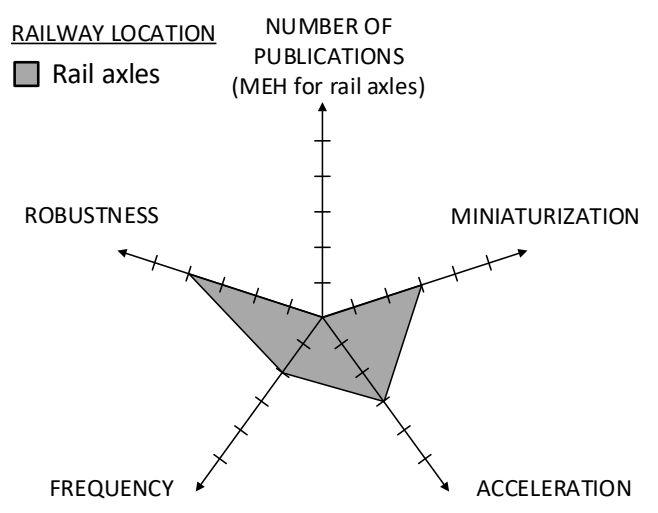

Fig. 15. Axles parameters analysis

\section{B. Rotational motion MEH taxonomy application}

After analyzing rail axles parameters profile, rotational motion MEH taxonomy is now applied to select the MEHT that is more appropriate to convert axle's rotational motion into electric energy, in order to power WSNs placed in such location. Every energy harvesting system placed on rail axles must be robust in order to resist harsh conditions and high speeds experienced in this location. As exposed in Fig.16 and Fig.17, electromagnetic, variable reluctance and Wiegand technologies meet robustness requirements while the rest of MEHTs are not robust enough to be used in rail axles. As a consequence of the severe space constraints on axles, the energy harvesting system must have a high miniaturization parameter value. Despite the fact that all MEHTs meet miniaturization requirements to be applied in rail car axles, electrostatic, Wiegand and magnetoelectric technologies are the most appropriate to be miniaturized.

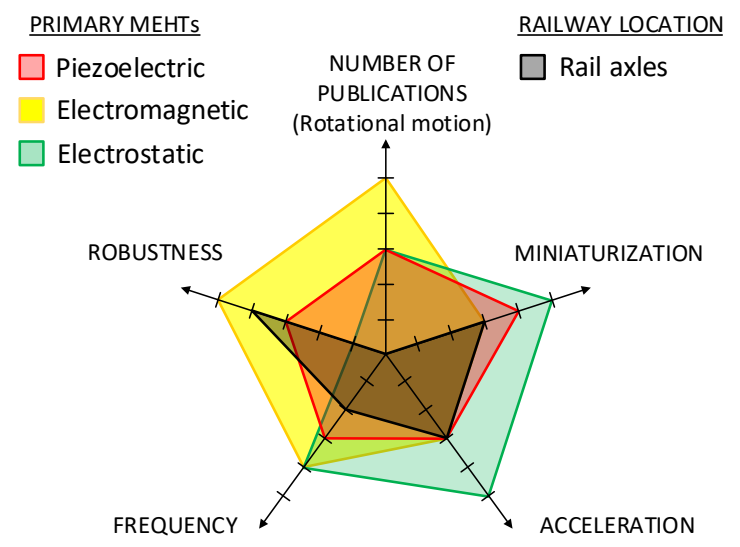

Fig. 16. Primary MEHTs compatibility with rail axles

Analyzing the frequency and acceleration ranges of each MEHT, it can be concluded that all MEHT can collect the rotation frequencies while it can be also observed that all MEHTs, except magnetoelectric and Wiegand, are capable to collect 10's G accelerations of analyzed location. 


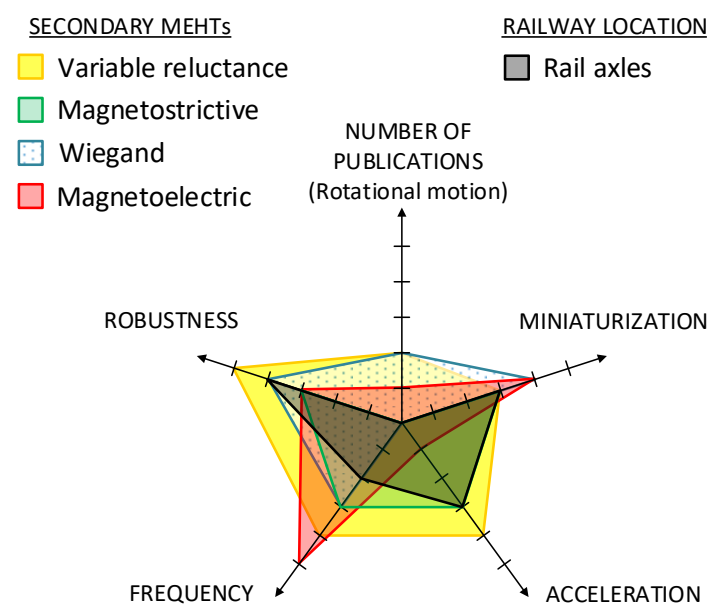

Fig. 17. Secondary MEHTs compatibility with rail axles

Thus, it can be concluded that electromagnetic technology is the most appropriate MEHT due to high robustness, technology maturity and efficiency on harsh environments at high speeds. Variable reluctance can be also a good MEHT to apply on rail axles due to the variation of reluctance that can be found in a railway environment and the good capability that this technology has to be used in harsh environments. Finally, Wiegand technology can be applied as a secondary energy harvesting system used to support other MEHTs. It can be used to recover energy when axles rotate at low frequencies, where neither electromagnetic nor variable reluctance work properly as explained in Section II-B.

Furthermore, the rest of MEHTs are not considered appropriate to be located in rail car axles because they do not meet the necessary robustness requirements. As soon as this problem is solved, they will become relevant MEHTs to convert rotational motion present in the axles into electric energy.

\section{Conclusion}

The predicted expansion of WSNs use due to IIoT will need all energy harvesting knowledge to be structured in order to facilitate its implementation as WSNs power source. A systematic review of MEHSs and MEHTs state of the art is performed in order to develop a comprehensive taxonomy. A comprehensive procedure to taxonomize this knowledge has been developed analyzing key parameters based on existing bibliography. The taxonomy performed is applied to evaluate primary and secondary MEHTs for rotational motion as MEHS.

In order to demonstrate the industrial applications of MEH taxonomy, it is applied to the railway industry, concluding that electromagnetic, variable reluctance and Wiegand are the most appropriate MEHTs to convert rotational motion of rail axles into electric energy in order to power AESSs placed there.

Thus, the taxonomy performed allows to identify the MEHT that is more appropriate for each rotary application, research gaps and upcoming challenges in the field of MEH applied to rotational motion. It also allows to analyze the compatibility between MEHTs in order to create hybrid harvesters which would allow to convert as much mechanical energy as possible into electric power in an industrial environment.

\section{REFERENCES}

[1] P. López Díez, I. Gabilondo, E. Alarcón, and F. Moll, “A Comprehensive Method to Taxonomize MEH Technologies,” in IEEE International Symposium on Circuits \& Systems (ISCAS), Florence, Italy, May 2018.

[2] L. D. Xu, W. He, S. Li, L. Da Xu, W. He, and S. Li, "Internet of things in industries: A survey," IEEE Transactions on Industrial Informatics, vol. 10, no. 4, pp. 2233-2243, 2014

[3] R. J. M. Vullers, R. Van Schaijk, I. Doms, C. V. Hoof, and R. Mertens, "Micropower energy harvesting," Solid State Electronics, vol. 53, pp. 684-693, 2009.

[4] H. A. Sodano, D. J. Inman, and G. Park, "A review of power harvesting from vibration using piezoelectric materials," Shock and Vibration Digest, vol. 36 , no. 3 , pp. $197-206,2004$

[5] R. Caliò, U. B. Rongala, D. Camboni, M. Milazzo, C. Stefanini, G. De Petris, and C. M. Oddo, "Piezoelectric energy harvesting solutions," Sensors, vol. 14, no. 3, pp. 4755-4790, 2014.

[6] M. Penella and M. Gasulla, "A review of commercial energy harvesters for autonomous sensors," in Instrumentation and Measurement Technology Conference Proceedings. IMTC 2007. IEEE, 2007, pp. 1-5.

[7] S. Priya and D. J. Inman, Energy harvesting technologies. Springer, 2009, vol. 21.

[8] P. D. Mitcheson, E. M. Yeatman, G. K. Rao, A. S. Holmes, and T. C. Green, "Energy harvesting from human and machine motion for wireless electronic devices," Proceedings of the IEEE, vol. 96, no. 9, pp. 1457-1486, 2008.

[9] T. J. Kazmierski and S. Beeby, Energy harvesting systems. Springer, 2014.

[10] E. Aguirre, P. Lopez-Iturri, L. Azpilicueta, A. Redondo, J. J. Astrain, J. Villadangos, A. Bahillo, A. Perallos, and F. Falcone, "Design and implementation of context aware applications with wireless sensor network support in urban train transportation environments," IEEE Sensors Journal, vol. 17, no. 1, pp. 169-178, 2017. 
[11] P. Spies, M. Pollak, and L. Mateu, Handbook of energy harvesting power supplies and applications. CRC Press, 2015.

[12] R. Resnick, J. Walker, and D. Halliday, Fundamentals of physics. John Wiley, 1988, vol. 1.

[13] C. Siu, Q. Shen, and R. Milne, "A fuzzy expert system for vibration cause identification in rotating machines," in Proceedings of the Sixth IEEE International Conference on Fuzzy Systems, vol. 1. IEEE, 1997, pp. 555-560.

[14] T. Ikeda, Fundamentals of piezoelectricity. Oxford, 1990.

[15] J. J. Bernstein, J. Bottari, K. Houston, G. Kirkos, R. Miller, B. Xu, Y. Ye, and L. E. Cross, "Advanced MEMS ferroelectric ultrasound 2D arrays," in Ultrasonics Symposium, vol. 2. IEEE, 1999, pp. 1145-1153.

[16] K. Cook-Chennault, N. Thambi, and A. Sastry, "Powering mems portable devices - a review of non-regenerative and regenerative power supply systems with special emphasis on piezoelectric energy harvesting systems," Smart Materials and Structures, vol. 17, no. 4, p. 043001, 2008.

[17] T. Rödig, A. Schönecker, and G. Gerlach, "A survey on piezoelectric ceramics for generator applications," Journal of the American Ceramic Society, vol. 93, no. 4, pp. 901-912, 2010.

[18] Y. Yang, Q. Shen, J. Jin, Y. Wang, W. Qian, and D. Yuan, "Rotational piezoelectric wind energy harvesting using impact-induced resonance Rotational piezoelectric wind energy harvesting using impact-induced resonance," Applied Physics Letters, vol. 105, no. 5, p. 053901, 2014.

[19] P. Pillatsch, E. M. Yeatman, and A. S. Holmes, "Piezoelectric rotational energy harvester for body sensors using an oscillating mass," in IEEE International Conference on Wearable and Implantable Body Sensor Networks (BSN), 2012, pp. 6-10.

[20] R. Lockhart, P. Janphuang, D. Briand, and N. F. D. Rooij, "A wearable system of micromachined piezoelectric cantilevers coupled to a rotational oscillating mass for on-body energy harvesting," in IEEE 27th International Conference on Micro Electro Mechanical Systems (MEMS), 2014, pp. 370-373.

[21] P. Pillatsch, E. M. Yeatman, and A. S. Holmes, "A wearable piezoelectric rotational energy harvester," in IEEE International Conference on Body Sensor Networks (BSN), 2013, pp. 1-6.

[22] P. Janphuang, R. A. Lockhart, D. Isarakorn, S. Henein, D. Briand, and N. F. D. Rooij, "Harvesting energy from a rotating gear using an AFM-like MEMS piezoelectric frequency up-converting energy harvester," Journal of Microelectromechanical Systems, pp. 742-754, 2015.

[23] L. Gu and C. Livermore, "Passive self-tuning energy harvester for extracting energy from rotational motion," Applied Physics Letters, vol. 97, no. 8, p. 081904,2010

[24] P. Pillatsch, E. M. Yeatman, and A. S. Holmes, "Experimental Validation of a Piezoelectric Frequency Up-Converting Rotational Harvester," in 11th International Conference on Wearable and Implantable Body Sensor Networks (BSN), 2014, pp. 6-10.

[25] H. Fu and E. M. Yeatman, "A methodology for low-speed broadband rotational energy harvesting using piezoelectric transduction and frequency up-conversion," Energy, vol. 125, pp. 152-161, 2017.

[26] M.-O. Kim, Y. Oh, Y. Kang, K.-H. Cho, J. Choi, and J. Kim, "Flexible piezoelectric strain energy harvester responsive to multi-directional input forces and its application to self-powered motion sensor," in IEEE 30th International Conference on Micro Electro Mechanical Systems (MEMS). IEEE, 2017, pp. 37-40.

[27] W. Kim, J. Yoo, and D. Choi, "Output power dependence of piezoelectric nanogenerator under mechanical deformation," in Fifth Asia International Symposium on Mechatronics (AISM 2015), Guilin, China, 2015.

[28] M. Umeda, K. Nakamura, and S. Ueha, "Analysis of the transformation of mechanical impact energy to electric energy using piezoelectric vibrator," Japanese Journal of Applied Physics, vol. 35, no. 5S, p. 3267, 1996.

[29] E. Jacquelin, S. Adhikari, and M. I. Friswell, "A piezoelectric device for impact energy harvesting," Smart Materials and Structures, vol. 20, no. 10, p. $105008,2011$.

[30] M. Ferrari, F. Cerini, and V. Ferrari, "Autonomous sensor module powered by impact-enhanced energy harvester from broadband low-frequency vibrations," in Transducers \& Eurosensors XXVII: The 17th International Conference on Solid-State Sensors, Actuators and Microsystems, 2013, pp. $2249-2252$.

[31] D. A. Sanchez, J. Leicht, F. Hagedorn, E. Jodka, E. Fazel, and Y. Manoli, "A parallel-sshi rectifier for piezoelectric energy harvesting of periodic and shock excitations," IEEE Journal of Solid-State Circuits, vol. 51, no. 12, pp. 2867-2879, 2016.

[32] R. Guigon, J.-J. Chaillout, T. Jager, and G. Despesse, "Harvesting raindrop energy: experimental study," Smart Materials and Structures, vol. 17, no. 1, p. 015039,2008

[33] A. S. Holmes, F. Häggström, J. Gustafsson, and J. Delsing, "Energy harvesting technologies for wireless sensors in rotating environments," in IEEE Emerging Technology and Factory Automation (ETFA), no. 1, 2014, pp. 1-4.

[34] D. P. Arnold, "Review of microscale magnetic power generation," IEEE Transactions on Magnetics, vol. 43, no. 11, pp. 3940-3951, 2007.

[35] B. Lafarge, O. Curea, A. Hacala, and H. Camblong, "Analysis, design \& simulation of an electromechanical energy harvesting system using a linear movement," in International Conference on Green Energy, 2014, pp. 233-239.

[36] J. Willemin, S. Boisseau, L. Olmos, M. Gallardo, G. Despesse, and T. Robert, "20000g shock energy harvesters for gun-fired munition," in Journal of Physics: Conference Series, vol. 773, no. 1. IOP Publishing, 2016, p. 012084.

[37] A. Wicks, Shock \& Vibration, Aircraft/Aerospace, and Energy Harvesting. Springer, 2015.

[38] P. Li, L. Zuo, J. Lu, and L. Xu, "Electromagnetic regenerative suspension system for ground vehicles," in IEEE International Conference on Systems, Man and Cybernetics (SMC), 2014, pp. 2513-2518.

[39] R. Oprea, M. Mihailescu, A. Chirila, and I. Deaconu, "Design and efficiency of linear electromagnetic shock absorbers," in 13th International Conference on Optimization of Electrical and Electronic Equipment (OPTIM). IEEE, 2012, pp. 630-634.

[40] S. Roundy, P. K. Wright, and J. Rabaey, "A study of low level vibrations as a power source for wireless sensor nodes," Computer Communications, vol. 26, no. 11, pp. 1131-1144, 2003.

[41] S. Boisseau, G. Despesse, and B. A. Seddik, "Electrostatic conversion for vibration energy harvesting," Small-Scale Energy Harvesting, pp. 1-39, 2012.

[42] P. P. Basset, E. Blokhina, and D. Galayko, Electrostatic kinetic energy harvesting. John Wiley \& Sons, 2016.

[43] S. Wang, L. Lin, and Z. L. Wang, "Triboelectric nanogenerators as self-powered active sensors," Nano Energy, vol. 11, pp. 436-462, 2015.

[44] G. Zhu, J. Chen, T. Zhang, Q. Jing, and Z. L. Wang, "Radial-arrayed rotary electrification for high performance triboelectric generator." Nature communications, vol. 5, p. 3426, 2014.

[45] A. Ahmed, I. Hassan, M. Hedaya, T. Abo El-Yazid, J. Zu, and Z. L. Wang, "Farms of triboelectric nanogenerators for harvesting wind energy: A potential approach towards green energy," Nano Energy, vol. 36, pp. 21-29, jun 2017.

[46] L. Lin, S. Wang, S. Niu, C. Liu, Y. Xie, and Z. L. Wang, "Noncontact free-rotating disk triboelectric nanogenerator as a sustainable energy harvester and self-powered mechanical sensor," ACS Applied Materials and Interfaces, vol. 6, no. 4, pp. 3031-3038, 2014.

[47] L. Lin, S. Wang, Y. Xie, Q. Jing, S. Niu, Y. Hu, and Z. L. Wang, "Segmentally structured disk triboelectric nanogenerator for harvesting rotational mechanical energy," Nano Letters, vol. 13, no. 6, pp. 2916-2923, 2013.

[48] P. Bai, G. Zhu, Y. Liu, J. Chen, Q. Jing, W. Yang, J. Ma, G. Zhang, and Z. L. Wang, "Cylindrical rotating triboelectric nanogenerator," ACS Nano, vol. 7, no. 7, pp. 6361-6366, 2013.

[49] C. Han, C. Zhang, W. Tang, X. Li, and Z. L. Wang, "High power triboelectric nanogenerator based on printed circuit board (PCB) technology," Nano Research, vol. 8, no. 3, pp. 722-730, 2015.

[50] C. Jean-Mistral, M. Beaune, T. Vu-Cong, and A. Sylvestre, "Energy scavenging strain absorber: application to kinetic dielectric elastomer generator," in SPIE Smart Structures and Materials Nondestructive Evaluation and Health Monitoring. International Society for Optics and Photonics, 2014, pp. $90561 \mathrm{H}-90561 \mathrm{H}$. 
[51] H. Guo, M.-H. Yeh, Y.-C. Lai, Y. Zi, C. Wu, Z. Wen, C. Hu, and Z. L. Wang, "All-in-one shape-adaptive self-charging power package for wearable electronics," ACS nano, vol. 10, no. 11, pp. 10580-10 588, 2016.

[52] R. I. Haque, P.-A. Farine, and D. Briand, "Electrically conductive fabric based stretchable triboelectric energy harvester," in Journal of Physics: Conference Series, vol. 773, no. 1. IOP Publishing, 2016, p. 012005.

[53] O. Heaviside, Electrical papers. Cambridge University Press, 2011, vol. 2.

[54] D. Parthasarathy, P. Enoksson, and R. Johansson, "Prototype energy harvesting wheel speed sensor for anti-lock braking," in IEEE International Symposium on Robotic and Sensors Environments (ROSE), 2012, pp. 364-369.

[55] B. Yan, C. Zhang, L. Li, H. Zhang, and S. Deng, "Design and construction of magnetostrictive energy harvester for power generating floor systems," in 18th International Conference on Electrical Machines and Systems (ICEMS), 2015, pp. 409-412.

[56] L. Wang and Others, "Vibration energy harvesting by magnetostrictive material for powering wireless sensors," Master's thesis, North Carolina State University, 2008.

[57] H. Zhang, "Power generation transducer from magnetostrictive materials," Applied Physics Letters, vol. 98, no. 23, p. $232505,2011$.

[58] L. Wang and F. G. Yuan, "Vibration energy harvesting by magnetostrictive material," Smart Materials and Structures, vol. 17, pp. 45 009-14, 2008.

[59] V. Berbyuk and J. Sodhani, "Towards modelling and design of magnetostrictive electric generators," Computers and Structures, vol. 86, pp. 307-313, 2008.

[60] T. Ueno and S. Yamada, "Performance of energy harvester using iron-gallium alloy in free vibration," IEEE Transactions on Magnetics, vol. 47, no. 10, pp. 2407-2409, 2011.

[61] J.-H. Yoo and A. B. Flatau, "A bending-mode galfenol electric power harvester," Journal of Intelligent Material Systems and Structures, vol. 23, no. 6, pp. 647-654, 2012.

[62] B. Rezaeealam, T. Ueno, and S. Yamada, "Finite element analysis of galfenol unimorph vibration energy harvester," IEEE Transactions on Magnetics, vol. 48, no. 11, pp. 3977-3980, 2012.

[63] H.-A. Chin, T. Liang, S. Xu, G. R. Poirier, N. Yao, S. Wagner, and M. C. McAlpine, "Wireless biomechanical power harvesting via flexible magnetostrictive ribbons," Energy \& Environmental Science, vol. 7, no. 7, pp. 2243-2249, 2014

[64] J. Wiegand, “"Bistable Magnetic Device”, United States Patent 3,820,090," 1974.

[65] S. Saggini, F. Ongaro, L. Corradini, and A. Affanni, "Low-power energy harvesting solutions for wiegand transducers," IEEE Journal of Emerging and Selected Topics in Power Electronics, vol. 3, pp. 766-779, 2015.

[66] J. Wiegand, "'Method of manufacturing bistable magnetic device", United States Patent 3,892,118," 1975.

[67] R. Zentgraf and U. Bochtler, "Use case analysis of Wiegand-based energy harvester in mechanical sensing devices," in PIERS Proceedings, 2015, pp. $1580-1582$.

[68] M. I. Bichurin, V. M. Petrov, and R. V. Petrov, "Direct and inverse magnetoelectric effect in layered composites in electromechanical resonance range : A review," Journal of Magnetism and Magnetic Materials, vol. 324, no. 21, pp. 3548-3550, oct 2012.

[69] A. Lasheras, J. Gutiérrez, S. Reis, D. Sousa, M. Silva, P. Martins, S. Lanceros-Mendez, J. M. Barandiarán, D. A. Shishkin, and A. P. Potapov, "Energy harvesting device based on a metallic glass/PVDF magnetoelectric laminated composite," Smart Materials and Structures, vol. 24, no. 6, p. 65024, 2015.

[70] J. Ryu, A. V. Carazo, K. Uchino, and H.-E. Kim, "Magnetoelectric properties in piezoelectric and magnetostrictive laminate composites," Japanese Journal of Applied Physics, vol. 40, pp. 4948-4951, 2001

[71] X. Zhao and D. G. Lord, "Application of the Villari effect to electric power harvesting," Journal of Applied Physics, vol. 99, no. 8, pp. 10-13, 2006,

[72] X. Dai, Y. Wen, P. Li, J. Yang, and G. Zhang, "Modeling, characterization and fabrication of vibration energy harvester using terfenol-d/pzt/terfenol-d composite transducer," Sensors and Actuators A: Physical, vol. 156, no. 2, pp. 350-358, 2009.

[73] S. D. Moss, J. E. McLeod, I. Powlesland, and S. C. Galea, "Bi-axial vibration energy harvesting," Defense Science and Technology Organisation Victoria (Australia) Air Vehicles Division, Tech. Rep., 2012.

[74] S. D. Moss, J. E. McLeod, and S. C. Galea, "Wideband vibro-impacting vibration energy harvesting using magnetoelectric transduction," Journal of Intelligent Material Systems and Structures, vol. 24, no. 11, pp. 1313-1323, 2013.

[75] M. Li, Y. Wen, P. Li, J. Yang, and X. Dai, "A rotation energy harvester employing cantilever beam and magnetostrictive/piezoelectric laminate transducer," Sensors and Actuators, A: Physical, vol. 166, no. 1, pp. 102-110, 2011.

[76] M. Li, Y. Wen, P. Li, and J. Yang, "A resonant frequency self-tunable rotation energy harvester based on magnetoelectric transducer," Sensors and Actuators, A: Physical, vol. 194, pp. 16-24, 2013.

[77] E. M. Yeatman, "Energy harvesting from motion using rotating and gyroscopic proof masses," Proceedings of the Institution of Mechanical Engineers, Part C: Journal of Mechanical Engineering Science, vol. 222, no. 1, pp. 27-36, 2008.

[78] R. G. Cid-Fuentes, M. Y. Naderi, K. R. Chowdhury, A. Cabellos-Aparicio, and E. Alarcón, "On the scalability of energy in wireless rf powered internet of things," IEEE Communications Letters, vol. 20, no. 12, pp. 2554-2557, 2016.

[79] S. D. Moss, O. R. Payne, G. A. Hart, and C. Ung, "Scaling and power density metrics of electromagnetic vibration energy harvesting devices," Smart Materials and Structures, vol. 24, no. 2, p. 023001, 2015.

[80] E. Ciravegna, E. Alarcón, F. Moll, and E. Macii, “Design of an efficient power management integrated circuit for kinetic energy harvesters," Master's thesis, Universitat Politècnica de Catalunya, 2015.

[81] S. P. Beeby, M. J. Tudor, and N. White, "Energy harvesting vibration sources for microsystems applications," Measurement Science and Technology, vol. 17, no. 12, p. R175, 2006.

[82] P. D. Mitcheson, E. K. Reilly, T. Toh, P. K. Wright, and E. M. Yeatman, "Performance limits of the three mems inertial energy generator transduction types," Journal of Micromechanics and Microengineering, vol. 17, no. 9, p. S211, 2007.

[83] Y. Tadesse, S. Zhang, and S. Priya, "Multimodal energy harvesting system: piezoelectric and electromagnetic," Journal of Intelligent Material Systems and Structures, vol. 20, no. 5, pp. 625-632, 2009.

[84] Y.-J. Wang, C.-D. Chen, and C.-K. Sung, "Design of a frequency-adjusting device for harvesting energy from a rotating wheel," Sensors and Actuators A: Physical, vol. 159, no. 2, pp. 196-203, 2010.

[85] E. Blokhina, A. El Aroudi, E. Alarcón, and D. Galayko, Nonlinearity in Energy Harvesting Systems. Springer, 2016.

[86] I. Sari, T. Balkan, and H. Kulah, "An electromagnetic micro power generator for wideband environmental vibrations," Sensors and Actuators A: Physical, vol. 145 , pp. 405-413, 2008.

[87] Z. L. Wang, "Triboelectric nanogenerators as new energy technology for self-powered systems and as active mechanical and chemical sensors," ACS nano, vol. 7, no. 11, pp. 9533-9557, 2013.

[88] T. T. Toh, P. D. Mitcheson, A. S. Holmes, and E. M. Yeatman, “A continuously rotating energy harvester with maximum power point tracking," Journal of Micromechanics and Microengineering, vol. 18, no. 10, p. 104008, 2008.

[89] A. S. Holmes, G. Hong, and K. R. Pullen, "Axial-flux permanent magnet machines for micropower generation," Journal of Microelectromechanical Systems, vol. 14, no. 1, pp. 54-62, 2005.

[90] Y. J. Wang, Y. T. Hao, and H. Y. Lin, "Design of a weighted-rotor energy harvester based on dynamic analysis and optimization of circular halbach array magnetic disk," Micromachines, vol. 6, no. 3, pp. 375-389, 2015.

[91] H. Raisigel, O. Cugat, and J. Delamare, "Permanent magnet planar micro-generators," Sensors and Actuators, A: Physical, vol. 130, pp. 438-444, 2006.

[92] D. A. Howey, A. Bansal, and A. S. Holmes, "Design and performance of a centimetre-scale shrouded wind turbine for energy harvesting," Smart Materials and Structures, vol. 20, no. 8, p. 85021, 2011. 
[93] X. H. Li, C. B. Han, T. Jiang, C. Zhang, and Z. L. Wang, "A ball-bearing structured triboelectric nanogenerator for nondestructive damage and rotating speed measurement," Nanotechnology, vol. 27, no. 8, p. 085401, 2016.

[94] P. Pillatsch, E. M. Yeatman, and A. Holmes, "Piezoelectric rotational energy harvester for body sensors using an oscillating mass," in International Conference on Wearable and Implantable Body Sensor Networks (BSN). IEEE, 2012, pp. 6-10.

[95] T. Xue, X. Ma, C. Rahn, and S. Roundy, "Analysis of upper bound power output for a wrist-worn rotational energy harvester from real-world measured inputs," in Journal of Physics: Conference Series, vol. 557, no. 1. IOP Publishing, 2014, p. 012090.

[96] P. Pillatsch, E. M. Yeatman, and A. Holmes, "A wearable piezoelectric rotational energy harvester," in IEEE International Conference on Body Sensor Networks (BSN). IEEE, 2013, pp. 1-6.

[97] T. T. Toh, P. D. Mitcheson, and E. M. Yeatman, "Continuously rotating energy harvester with improved power density," in Proceedings of PowerMEMS, 2008.

[98] T. T. Toh, a. Bansal, G. Hong, P. D. Mitcheson, A. S. Holmes, and E. M. Yeatman, "Energy harvesting from rotating structures," Technical Digest PowerMEMS 2007, pp. 327-330, 2007.

[99] N. Achotte, P. A. Gilles, O. Cugat, J. Delamare, P. Gaud, and C. Dieppedale, "Planar brushless magnetic micromotors," Journal of Microelectromechanical Systems, vol. 15, no. 4, pp. 1001-1014, 2006.

[100] D. Spreemann, Y. Manoli, B. Folkmer, and D. Mintenbeck, "Non-resonant vibration conversion," Journal of Micromechanics and Microengineering, vol. 16, no. 9, p. S169, 2006

[101] E. Romero, R. O. Warrington, and M. R. Neuman, "Body motion for powering biomedical devices," in International Conference of the IEEE Engineering in Medicine and Biology Society (EMBC), 2009, pp. 2752-2755.

[102] Y.-J. Wang and Y.-T. Hao, "Harvesting energy from ship rolling using an eccentric disk revolving in a hula-hoop motion," in IEEE International Power Electronics Conference (IPEC), 2014, pp. 1420-1424.

[103] M. Niroomand and H. R. Foroughi, "A rotary electromagnetic microgenerator for energy harvesting from human motions," Journal of Applied Research and Technology, vol. 14, no. 4, pp. 259-267, 2016.

[104] Q. Zhang, L. Gu, K. Yang, M. A. Halim, R. Rantz, and S. Roundy, "Kinetic energy harvesting using improved eccentric rotor architecture for wearable sensors," in IEEE Sensors, 2016, pp. 1-3.

[105] M. Halim, R. Rantz, Q. Zhang, L. Gu, K. Yang, and S. Roundy, "Electromagnetic energy harvesting from swing-arm motion using rotational eccentric mass structure," in 19th IEEE International Conference on Solid-State Sensors, Actuators and Microsystems (Transducers), 2017, pp. 1863-1866.

[106] D. Parthasarathy, "Energy harvesting wheel speed sensor," Master's thesis, Chalmers University of Technology, 2012.

[107] M. Kroener, S. K. T. Ravindran, and P. Woias, "Variable reluctance harvester for applications in railroad monitoring," in Journal of Physics: Conference Series, vol. 476. IOP Publishing, 2013, p. 012091.

[108] M. Kroener, N. Moll, S. K. T. Ravindran, P. Mehne, and P. Woias, "Characterization of a variable reluctance harvester," in Journal of Physics: Conference Series, vol. 557. IOP Publishing, 2014, p. 012035.

[109] F. Häggström, "Energy harvesting for smart-internet-connected bearings," Ph.D. dissertation, Luleå tekniska universitet, 2015.

[110] M. E. Staley and A. B. Flatau, "Characterization of energy harvesting potential of terfenol-d and galfenol," in Proc. of SPIE Vol, vol. 5764, 2005, p. 631.

[111] J. Wiegand, “'Pulse Generation by changing magnetic field", United States Patent 4,309,628," 1982.

[112] J. Wiegand and J. E. Opie, "'Pulse generator with shaped magnetic field", United States Patent 4,484,090," 1984

[113] F. Ongaro, S. Saggini, and L. Corradini, "Low-power energy harvester for wiegand transducers," in IEEE Applied Power Electronics Conference and Exposition (APEC), 2013, pp. 453-459.

[114] S. Dong, J. Zhai, J. Li, D. Viehland, and S. Priya, "Multimodal system for harvesting magnetic and mechanical energy," Applied Physics Letters, vol. 93, no. 10, p. 103511, 2008.

[115] V. J. Hodge, S. O'Keefe, M. Weeks, and A. Moulds, "Wireless sensor networks for condition monitoring in the railway industry: A survey," IEEE Transactions on Intelligent Transportation Systems, vol. 16, no. 3, pp. 1088-1106, 2015.

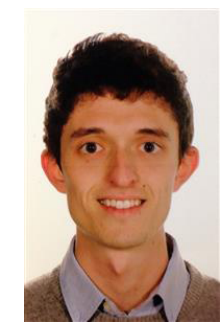

Pablo López Díez received the University Degree in Electronics and Industrial Automation by the University of León, Spain, in 2014, and the equivalent of the M.Sc. degree in Renewable Energy by the European University of Madrid, Spain, in 2016. As $\mathrm{PhD}$ candidate he investigates energy harvesting technologies for industrial applications at the Microsystems Team at IK4-Ikerlan, co-tutored by the Technical University of Catalunya (UPC), Spain.

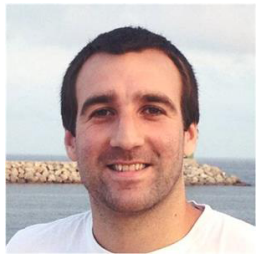

Iosu Gabilondo received his M.Sc. degree in Telecommunications Engineering at Mondragón University (Spain) in 2009. He started his professional activity at IK4-Ikerlan in 2010, where he is currently heading the Microsystems Team. He has participated and coordinated various industry focused projects, addressing electronic systems' design and development (HW \& SW). His main research activities include Smart Systems' Integration and electronic circuit design and development. 


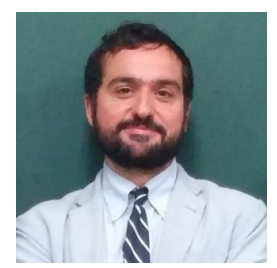

Eduard Alarcón received the M.Sc. (National Award) and Ph.D. degrees (Hons.) in electrical engineering from the Technical University of Catalunya (UPC BarcelonaTech), Barcelona, Spain, in 1995 and 2000, respectively. Since 1995, he has been in the Department of Electronic Engineering, UPC, where he became an Associate Professor in 2000. He has co-authored more than 250 scientific publications, four books, four book chapters and four patents, and has been involved in different National, European, and USA (DARPA,NSF) R\&D projects. His research interests include on-chip energy management circuits, energy harvesting and wireless energy transfer, and nanotechnology-enabled wireless communications.

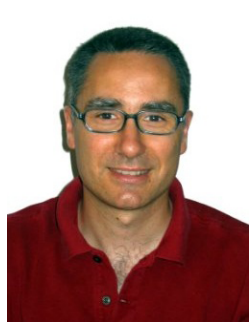

Francesc Moll received the equivalent of the M.Sc. degree in physics from the University of Balearic Islands, Palma, Spain, in 1991, and the Ph.D. degree in electronic engineering from the Technical University of Catalonia (UPC), Barcelona, Spain, in 1995. Since 1991, he has been with the Department of Electronic Engineering, UPC, where he is currently an Associate Professor. His current research interests include methods for energy harvesting oriented to low-power microelectronic circuits. 